\title{
THE DESIGN OF AN INTELLIGENT TUTOR SYSTEM BASED ON ADAPTIVE WORKFLOW
}

\author{
Mohammed Beyyoudh ${ }^{1}$, Mohammed Khalidi Idrissi ${ }^{2}$, Samir Bennani ${ }^{3}$
}

\begin{abstract}
This paper start a conception of Intelligent Tutor System (ITS) based on an adaptive workflow. The main idea of this work is the integration of workflow engine in the global architecture of ITS that allows an intelligent generation of process models and theirs executions in order to product an adaptive learning process as well as an adaptive pedagogical sequence. Our adaptation model use parameters provided by the adopted learner model especially student knowledge and preferences and teaching domain schema to propose the optimal sequence for each learner. The work presented in this article affords also the main lines for an intelligent tutor system supporting the coexistence of system and human tutor.

Keywords - Adaptive Workflow, Intelligent Tutor System, Adaptive Pedagogical Sequence, Learner Model, Teaching

Domain, Adaptation Model, Meta-model of process.
\end{abstract}

\section{INTRODUCTION}

The stunning technological development known in recent decades has pushed researchers in the field of education and teaching to concentrate their knowledge in the exploitation of IT in the automation of learning. Some works have focused on the use of artificial intelligence techniques for the individualization of learning through automated tutors. The idea is to equip the machine with human skills in order to have teaching expertise especially in the study fields (expert field), learning methods and the assessment of skills and knowledge of the learners. It is about designing intelligent systems with adaptable interfaces that allow learners to carry out educational activities that are part of the artificial learning process. The majority of the solutions adopted focus on the learning content and neglect the learning processes. In other words, how can we describe an effective and resulting distance education in the absence of a human tutor? The main objectives of intelligent tutoring systems are the intelligent provision of the right activity and pedagogical content at the right time to a learner, taking into account his knowledge, ie the dynamic definition of learning sequences or teaching scenarios. Hence the importance of introducing the notion of dynamic and adaptive workflow in an intelligent tutors system. It is for these purposes that we have explored the feasibility of such a system.

In this article, we begin to design a system combining the existence of an intelligent tutor and a human tutor, then we try to integrate a workflow management system into the global ITS architecture that allows to follow the execution of adaptive workflow instances in order to use them in the support of self-learning process models and the generation of adapted learning sequences.

In Section 1, we discuss the notion of adaptive workflows through the conception of a meta-model of the process. We also set up a model of the self-learning process that will be implemented in our system and that serves as for the learner's good follow-up by the human tutor. In Section 2, we describe the student model that we want to adopt for our case and that is built on the skills and preferences of the learner. Section 3 will be devoted to the description of the teaching domain model adopted. As for section 4, we present our model of adaptation of the pedagogical sequence to the competences of the learner based on the adaptive workflows. We will conclude with a synthesis summarizing all the works presented as well as the research perspectives.

\section{Terminology:}

We begin by defining the following concepts:

Intelligent Tutor System: Several definitions and architectures of these systems have been developed. Based on the definitions cited in [1], they are systems based on artificial intelligence techniques that automate the interventions of a tutor in the teaching process and are based on the following ingredients: The teaching field, the learner presentation, pedagogical

\footnotetext{
${ }^{1}$ RIME TEAM-Networking, Modeling and e-Learning- LRIE Laboratory- Research in Computer Science and Education Laboratory Mohammadia School Engineers (EMI) - Mohammed Vth University Rabat AV. Ibn Sina Agdal Rabat BP. 765 Morocco

2 RIME TEAM-Networking, Modeling and e-Learning- LRIE Laboratory- Research in Computer Science and Education Laboratory Mohammadia School Engineers (EMI) - Mohammed Vth University Rabat AV. Ibn Sina Agdal Rabat BP. 765 Morocco

3 RIME TEAM-Networking, Modeling and e-Learning- LRIE Laboratory- Research in Computer Science and Education Laboratory Mohammadia School Engineers (EMI) - Mohammed Vth University Rabat AV. Ibn Sina Agdal Rabat BP. 765 Morocco
} 
scenarios and the personalization of learning to the needs of the learners. The general architecture of such a system is generally based on 4 main modules: the domain model, the student model, the pedagogical expert, the learning interface.

The work on these systems focuses on the personalization of a learning sequence, pedagogical content and learning interfaces.

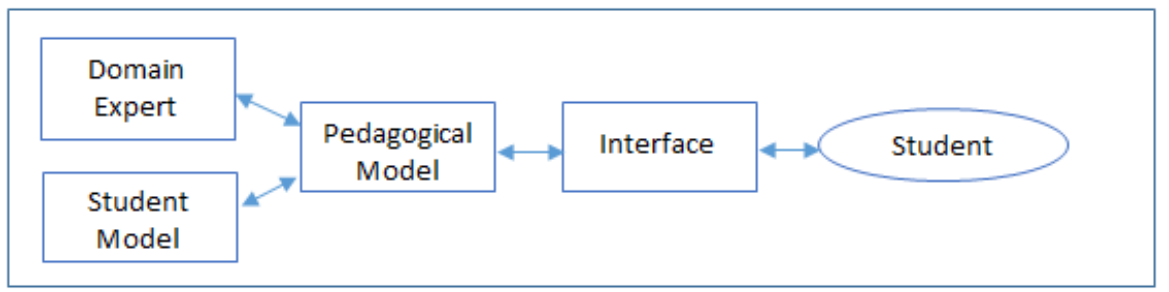

Figure 1: General model of an intelligent tutor proposed in [1]

The process as defined by WfMC in [2]: is a sequencing of activities or tasks ordered by transitions and performed by actors, whose goal is to achieve an objective. Each process has a start and an end. The actors can be human or software. The notion of workflow refers to the sequential computer representation of this process in which the execution of a task or an operation can generate a flow of data (information, documents, etc.).

By misuse of language, we confuse in this article a process and a workflow.

A workflow engine: is a software component that allows you to run workflow instances.

A workflow management system: is a system that integrates one or more workflow engines to execute one or more process instances, and also allows tracking and tracing of a running instance.

\section{META-MODEL OF THE AUTOMATIC LEARNING PROCESS:}

Workflows are usually used in information systems for business process definition. These, after their definitions, are automated through the execution of their models in a workflow engine. The notion of workflows is rarely used in e-learning platforms and especially in intelligent tutor systems. This lack of use is due to the lack of flexibility in the execution of the models and the possibility of their adaptations. These defects seem to be overcome by the introduction of the notion of adaptive workflow which is defined by a process that can be adapted as it is executed by introducing new tasks, deleting others, making loops on a sub -processes etc.

In this section, we draw up a state-of-the-art of e-learning platform based on workflow, then we define a meta-model of the process inspired by the work presented and we end up modeling the Self-learning workflow process that we propose to be used in our intelligent tutor system.

In [3] and [4], the authors propose a meta-modeling of the process based on the definitions adopted by the Workflow Management Coalition (WfMC). Found in [3] a meta-model drawn up according to 4 components:

- An organizational component: describes the organizational structures, the actors of the system and their roles.

- A functional component: describes the functionalities of the system.

- A behavioral component: describes tasks and control flows.

- An information component: describes the information system part allowing the completion of the work.

In [4], the authors design a meta-model supporting the use of the notion of flexible workflows in an open training context.

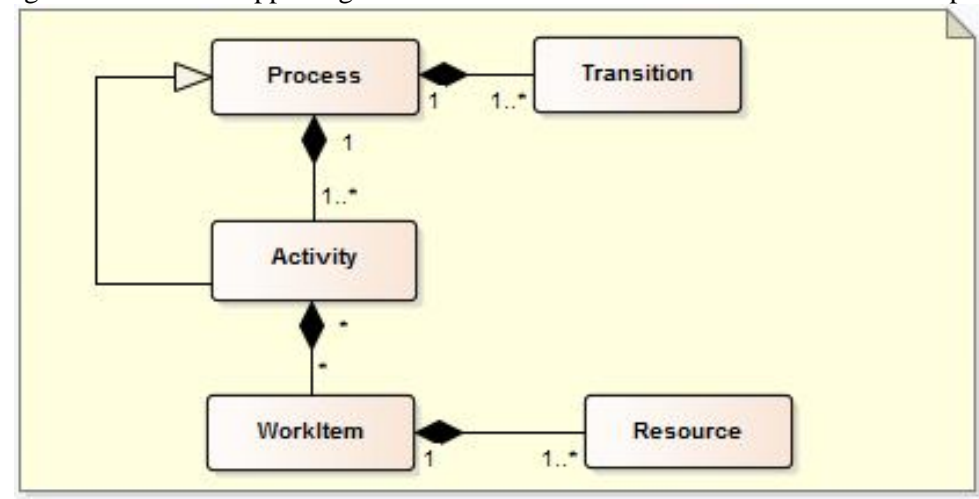

Figure 2: Meta-model of the process presented in [4]

Inspired from the work [3] and [4], we propose to define a meta-model of the process that allows expressing the models and which is based, in addition to the definition quoted in the part terminology, on the rules presented below: 
A process can consist of one or more sub processes. The process and sub-processes can be broken down into one or more tasks or activities ordered by transitions triggered by rules and conditions. A task, with the exception of the first and last of the process, has one or more subsequent and previous tasks, which allows to define the sequencing of the process. A task (activity) is carried out by the actors of the process, which can be a physical person, a group of individuals or a machine (system).

A task is the most basic element in a process (indecomposable atomic unit). A task generates or uses one or more resources (documents, form, interface ...).

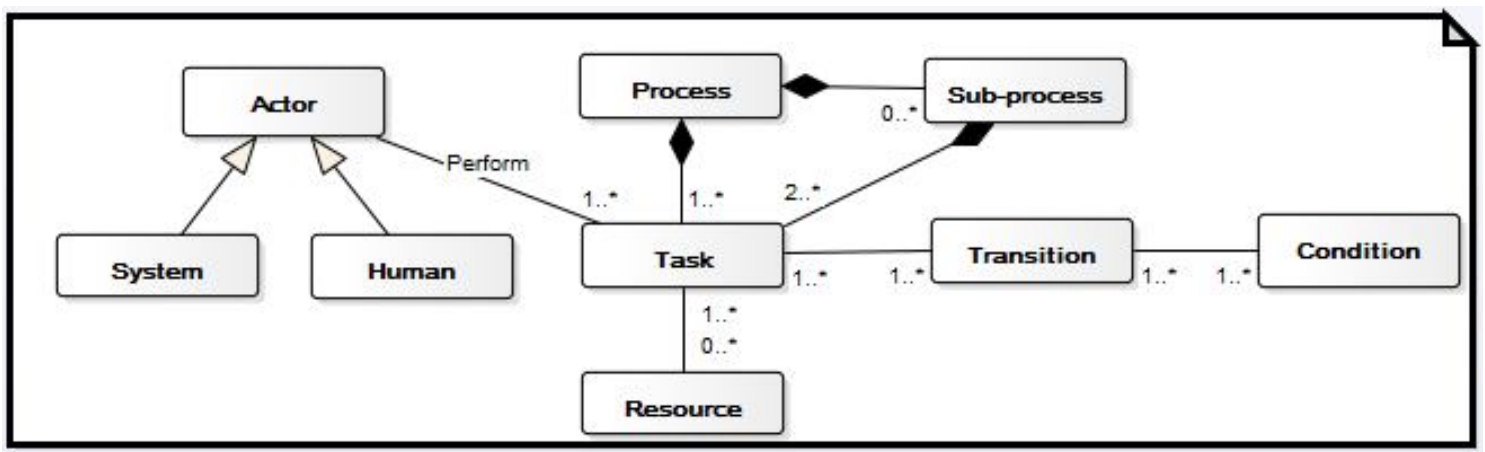

Figure 3: Meta-model of the process

The meta-model of the process thus defined makes it possible to construct workflow models that can be adapted according to the parameters passed in the rules or the conditions of transitions between the activities, this allows the intelligent production of the workflows which will serve us for adaptation of learning sequences to the learner profile (Adaptation model: section 4 of this article).

\section{Description of the model of the self-learning process adopted:}

The description of the automatic learning process is a very important element in understanding the process and the activities performed by each of its actors. In this part, we answer the question: how can we describe the self-learning process in a smart system?

Our goal is to design a self-learning system that combines the presence of an intelligent tutor that allows the automation of learning process activities and a human tutor who performs certain tasks including communication with the learner in case of need, content management, management of tasks base...

Our vision declines from the assumption which stipulates that whatever the intelligence of the system, there are always situations that require human intervention especially those that emphasize a boredom blocking which infect the learning interest of the learner. Following this hypothesis, we opt for the design of an intelligent system that involves the human tutor to ensure control, monitoring, management and the release of paralyzing situations.

Hence the idea of proposing the modeling of a learning process introducing 3 main actors: the learner, the system and the human tutor.

The process model, shown in Figure 4, is triggered when a learner attempts to use the system. For a new learner, the system proposes a questionnaire in which the learner initializes his profile, this questionnaire contains several pieces of information allowing constructing a global idea on the model of the learner, in particular his knowledge, his style of learning and his level (Learning path). For an already existing learner, the system identifies it through its authentication information (Email, username, password ...) shown in the Personal Information block presented in the Learner Model in the following section, the system then recognizes its model which contains all the information necessary for its educational path. Based on the learner's knowledge (acquired concepts), his pedagogical background (level of learning) and his preferences, the system proposes an adapted pedagogical sequence which contains a sequence of activities to achieve a pedagogical objective (learning of one or more concepts). Before the generation of the pedagogical content (course, application exercise, example, explanation ...) adapted to the learner's preferences and the proposed sequence, the student is required to perform a pre-test at the beginning of each sequence to test the Prerequisites for the smooth running of the pedagogical sequence. At the end of this pre-test, the learner must obtain a score superior to a certain threshold in order to be able to access the pedagogical content adapted to the proposed pedagogical sequence, if necessary the system proposes a new pedagogical sequence to assimilate the concepts constituting the prerequisites. At the end of the pedagogical sequence, the system performs a summative evaluation to ensure that the pedagogical objective is achieved. If the result obtained does not exceed a minimum score, the system records the current state of the learner for that proposed sequence and proposes to re-examine it by readjusting the teaching material, otherwise the system update Learner Model With all the information changed and the process is completed. 
The Human Tutor intervenes in the execution of some tasks, namely the management of the pedagogical content, the provision of explanations in case of student blocking. If the learner fails to achieve the objective through the various existing teaching resources, the student may request the intervention of a teacher using videoconferencing in order to complete the sequence in question.

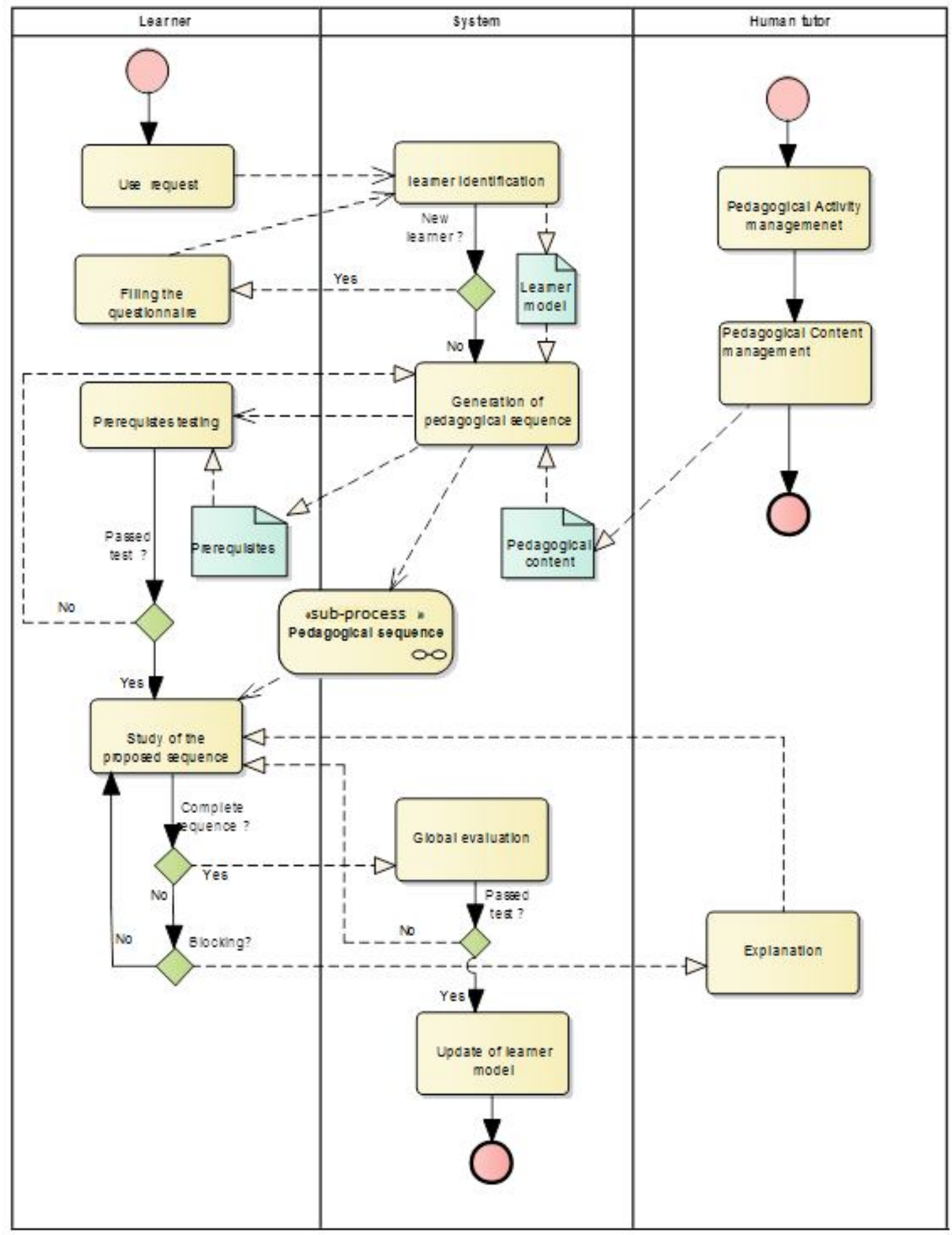

Figure 4: Model of self-learning process used

This automatic learning workflow will be implemented in our intelligent system in order to ensure a proper follow-up of the learner by the human tutor who intervenes in the case of a possible blockage. The system generates an instance of this workflow for each student who starts a learning session (interaction with the system). 


\section{LEARNER MODEL:}

Learner modeling is an indispensable component in the realization of intelligent tutor systems. It provides a comprehensive view of the different knowledge and characteristics of the learner. The student's model is usually exploited to: customize learning, assess learner knowledge and track his progress. In this article, we use the Learner Model to adapt a learning sequence and learning content to the learner's knowledge and preferences. Several approaches have been adopted to establish a student model including those based on the cognitive theory of profile representation, most of them classify the learner model into 5 main classes: Personal Data, Characteristics Of the learner, Interaction (System \& student), State of learning and Knowledge of the learner [5].

In this section, we answer the following question: What parameters should be considered in learner modeling and how should they be used?

We propose a model that contains 4 main classes, each one contains parameter-value pairs. This choice, which differs from the other approaches by combining the last two categories cited above in a single named learner competence, is justified by the consolidation of information about the cognitive state of the learner in a minimal number of classes which will be exploited later in the personalization of the student learning. The following figure represents our proposed ontology of the Learner Model:

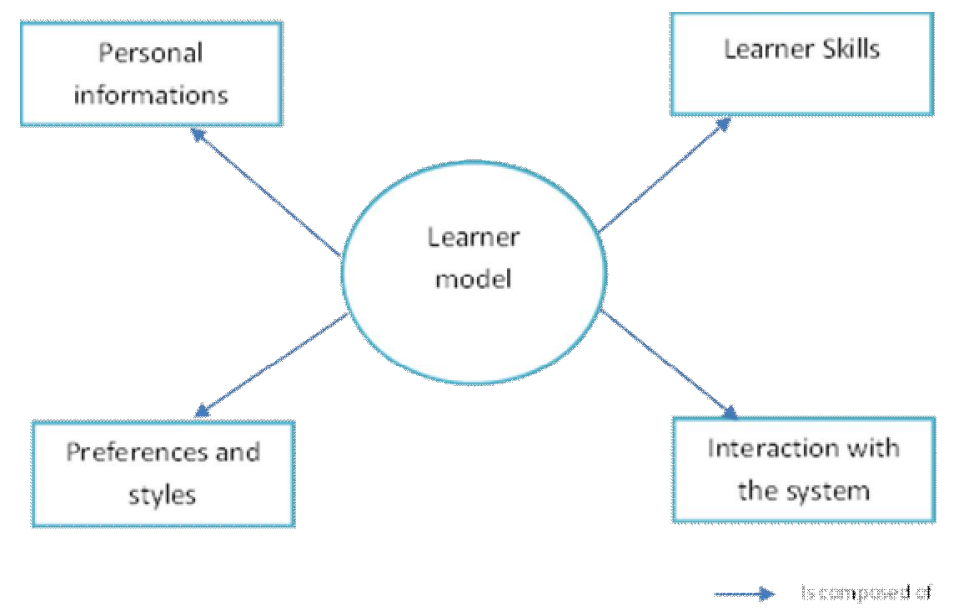

Figure 5: Ontology Model Learner Proposed

Personal Information: This class contains static data from the profile. It has general information about the learner. It contains attributes for identification and description of student characteristics such as name, first name, age, email address, gender, origin and nationality etc. This class will only be useful for the task of identifying the learner that is included in the self-learning process presented in the first section (figure 4).

Learner Skills (competences): This component is the most important component for adaptive education systems. It allows describing a learner in terms of his knowledge and his know-how in order to offer a personalized learning [17]. It contains the learner's pedagogical background, his level of learning, the acquired training, professional experiences, domain knowledge, acquired concepts, exam and test scores, pretest results, student beliefs, learning plans, learning objectives, frequent mistakes, etc.

Learner preferences: In this important component of the system, we introduced information about the learner's preferences and learning style, i.e., the psychological characteristics of the individual. It is made up of several parameters that allow us to describe what the learner prefers as didactic tools and methods to acquire knowledge. This model of preference is used to adapt learning systems to the students' desires, in particular the mode of acquisition of information (learning interface). In order to identify the psychological tendencies of a learner, several methods have been developed, especially Learning Style Theory [10], Index of Learning Styles ILS [9], the ISALEM (Inventory of Learning Styles, Multimedia Teaching).

Interaction with the system: This component of the learner's model makes it possible to keep a history of exchange of the student with the system and to trace the operations carried out throughout the learning process. The system can induce a learner's cognitive state from the recorded information. The information that we can use in this component is: the number of visits to pedagogical content, the type of teaching material, the duration of use of a resource, the time spent in learning, the number of explanations, the number of errors committed in a section, the number of correct answers, etc. The information acquired by each of these parameters makes it possible to draw one or more conclusions either on the learner or on the proposed content.

The parameters of each component of the adopted Learner Model will be used to accomplish a didactical activity in the process described in Section 1 as follows: 


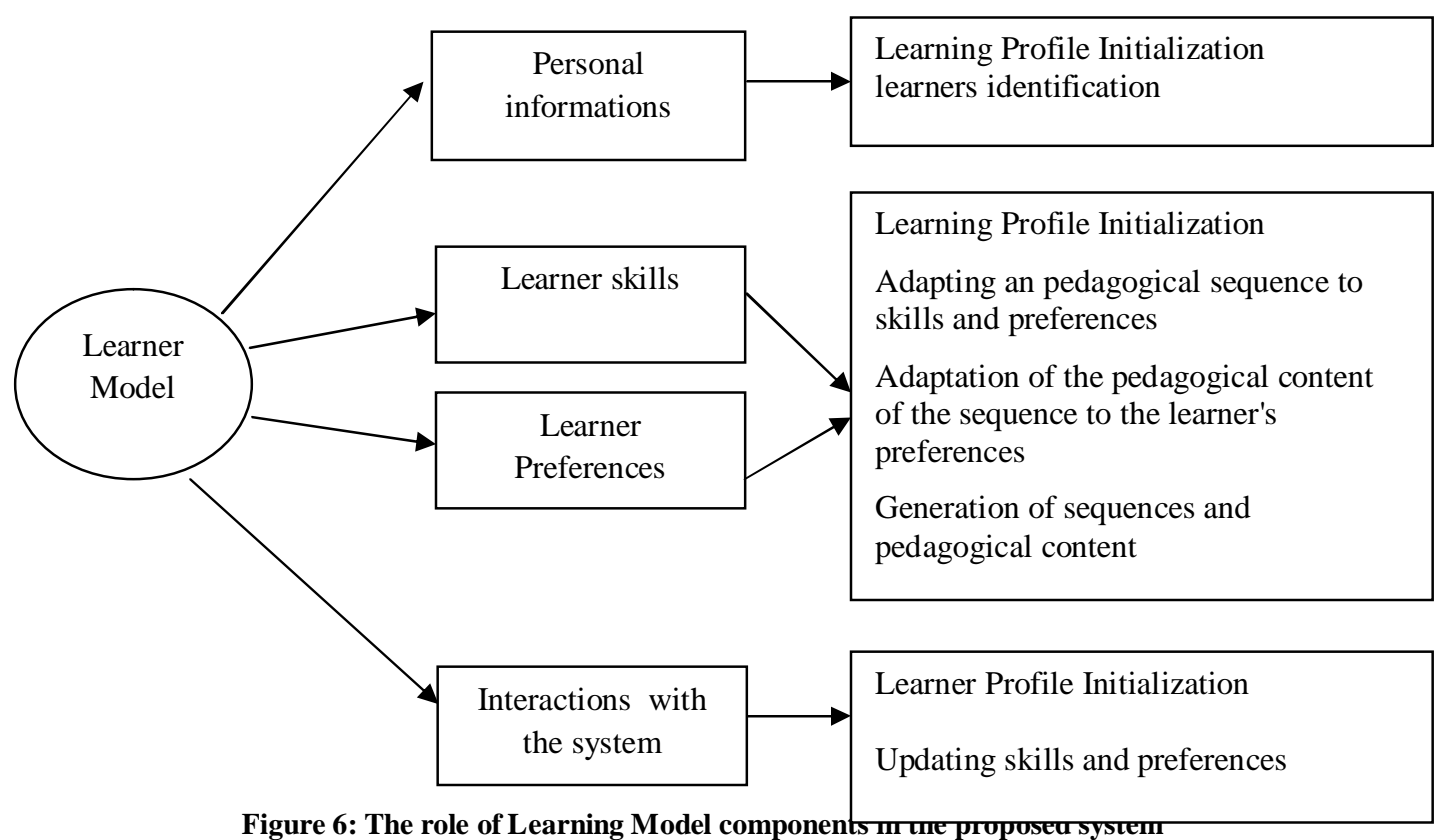

\section{Domain MODEL :}

The model of the teaching field is an indispensable component in learning especially when talking about intelligent tutor systems. This allows a decomposition of the teaching field into several elements in order to ensure a fine presentation of its content to the learner. This model, as specified in the work [15], can be described using two main elements:

- A schema of the domain that allows to describe, through a schematic presentation, the knowledge classes that constitute the domain, generally called Concepts [8], which are related by links connecting an origin and a destination [12], and which may be of different types such as "is composed of" "is sort of" [11].

- A Knowledge Base that contains the concept instances specified in the domain schema.

Several works have been devoted to the modeling of the domain as well as the formalisms of implementation of the models elaborated. For example, the MOT method "Modeling by Typed Objects" [11], CommonKADS [14] for modeling methods and Ontology, object-oriented models, semantic networks, neural networks for the formalisms of knowledge representation.

To our knowledge, the majority of the models in the domains studied are oriented towards the use of abstract concepts that do not take into account the notion of the didactic activities or tasks constituting the pedagogical sequences.

\section{Domain Schema:}

Our approach allows introducing a layer of pedagogical activities into our schema of the proposed domain, which will facilitate the implementation of our adaptation model based on adaptive workflows. Inspired by the work carried out in [6], we propose a decomposition of teaching domain into seven main concepts: The notions of teaching domain, concepts, learning objectives, pedagogical sequences, pedagogical activities, resources, potential errors.

The notions can be decomposed iteratively into several sub-notions until reaching the most granular level that contains concepts. Thus the concepts will represent the most elementary elements of knowledge (indecomposable). Each notion allows attaining a pedagogical objective by means of the learning of one or more pedagogical sequences which consist of several didactic activities (tasks). Each activity uses resources and teaching materials necessary for its execution. Each concept of the domain can be linked to a list of potential errors that can be committed by learners. It should be noted that the proposed schema can be extended later in terms of concepts mentioned.

The concepts mentioned above, will be related using different types of links: «Is a prerequisite for », «Is associated to », «Is linked to ». The following figure contains an UML modeling of the proposed teaching domain schema. 


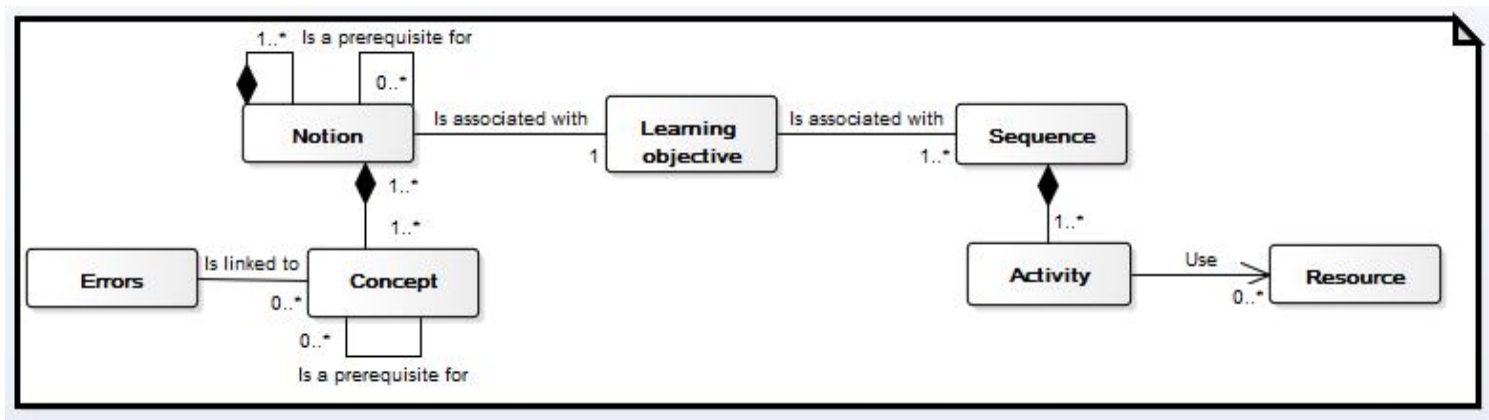

Figure 7: Class diagram of teaching domain

\section{Knowledge base:}

The knowledge base usually contains instances of the concepts used in the domain schema presented in a usable format. We advocate a hierarchical presentation, using a tree structure, the notions of the domain to be taught. Each node of the tree contains notions and sub-concepts linked by hierarchical links signifying the order of learning of each notion, the leaves of the tree being devoted to the lowest level elements which are the concepts (Figure 8).

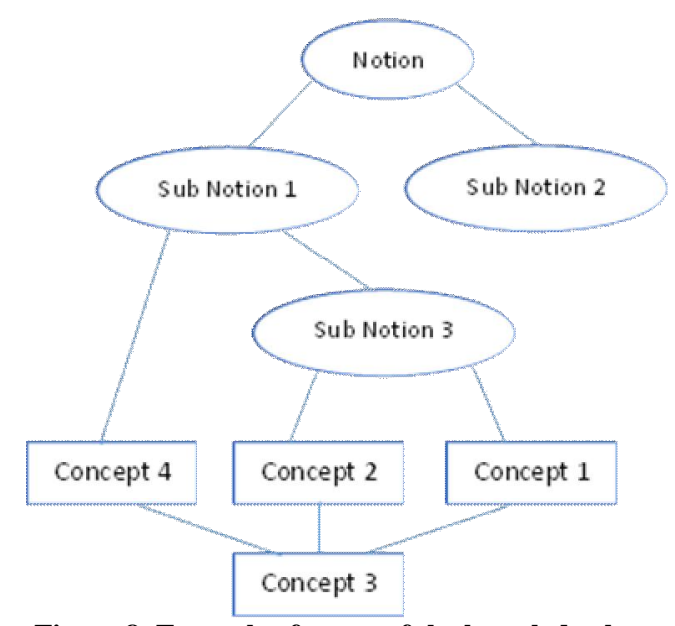

Figure 8: Example of a tree of the knowledge base

\section{AdAPTATION MODEL :}

In this section, we explain our strategy of adapting the pedagogical sequence to the learner's need for knowledge, through the exploration of the methods used the exposure of our technique which is based on the implementation of adaptive workflows and the architecture of the proposed system to respond to such a method.

The adaptation strategy in adaptive learning systems can be defined as the set of mechanisms and actions adopted by the system in order to adjust the learning path to each student taking into account his or her knowledge and preferences, it is based on the choice of the pedagogical sequence most relevant to achieve the determined objective. The work in this direction has highlighted the existence of two adaptation techniques: adaptation of the presentation and adaptation of navigation [13].

The adaptation of the presentation consists in displaying an interface adapted to the various characteristics of the student, ie the continuous modification of the appearance of the displayed content (level of detail, media used ...) to the user according to the evolution of the different cognitive aspects of the learner in order to reduce his learning load. Several techniques have been used for the implementation of this type of adaptation such as "Stretchtext" [7], "progressive access" [16].

The adaptation of navigation consists in orienting the learner in his pedagogical path by scheduling the different pedagogical objectives, based on the learner's state of knowledge in a field, with the aim of achieving these pedagogical objectives by following the most common path for each learner. Systems using this method recommend a hierarchical structure of the content. There are two types of techniques for the implementation of this adaptation mode: annotation of the link and scheduling of activities [13].

Our approach focuses on the adaptation of navigation with the technique of scheduling pedagogical activities. The idea is to make a decision on the choice of a pedagogical sequence that is most relevant for a learner based on the concepts already acquired by the student and his current level of learning. We consider a pedagogical sequence as an ordered sequence of 
activities whose aim is to achieve an objective (learning a notion, a concept...). This definition joins the one of the workflow. It is, therefore, proposed to consider a pedagogical sequence as a workflow. Our adaptation process begins by choosing a pedagogical sequence that is most relevant to the decision-making engine, taking as input parameters the student's level of learning, the concepts acquired in a domain and his general knowledge. These parameters will be taken from the learner model defined previously. The decision engine generates the necessary prerequisites for the good learning of each sequence, and then a sequence composer (figure: 9) performs composition of the optimal workflow of the sequence by identifying the pedagogical activities to be performed by the learner in the Tasks base as well as content adapted to the cognitive profile of the student in the content base. The composer also generates a pretest to be performed by the user before beginning the sequence learning as described in the proposed global process (figure 4). The workflow engine is responsible for running the instance if the pre-test is successful. The human tutor will be able to follow the execution of the pedagogical activities of the sequence using the workflow management system which makes it possible to visualize the current state of the execution. in case of an overflow in the execution time of a activity, the tutor may notice the blocking of the workflow and can take action to give explanations if necessary. The workflow can be adapted during its execution (adaptive workflow), that is to say it is possible to introduce new activities, change the order of tasks, delete a task, etc. This is based on the learner system interaction in the learner model. Indeed, our idea is to preconize partials evaluations for activities in which the system anticipates learning a concept, before doing the summative evaluation planned at the end of the sequence. An intelligent supervisor allows the student data to be updated as the workflow progresses, and returns this information to the decision engine in order to adapt the proposed sequence.

\section{System Architecture:}

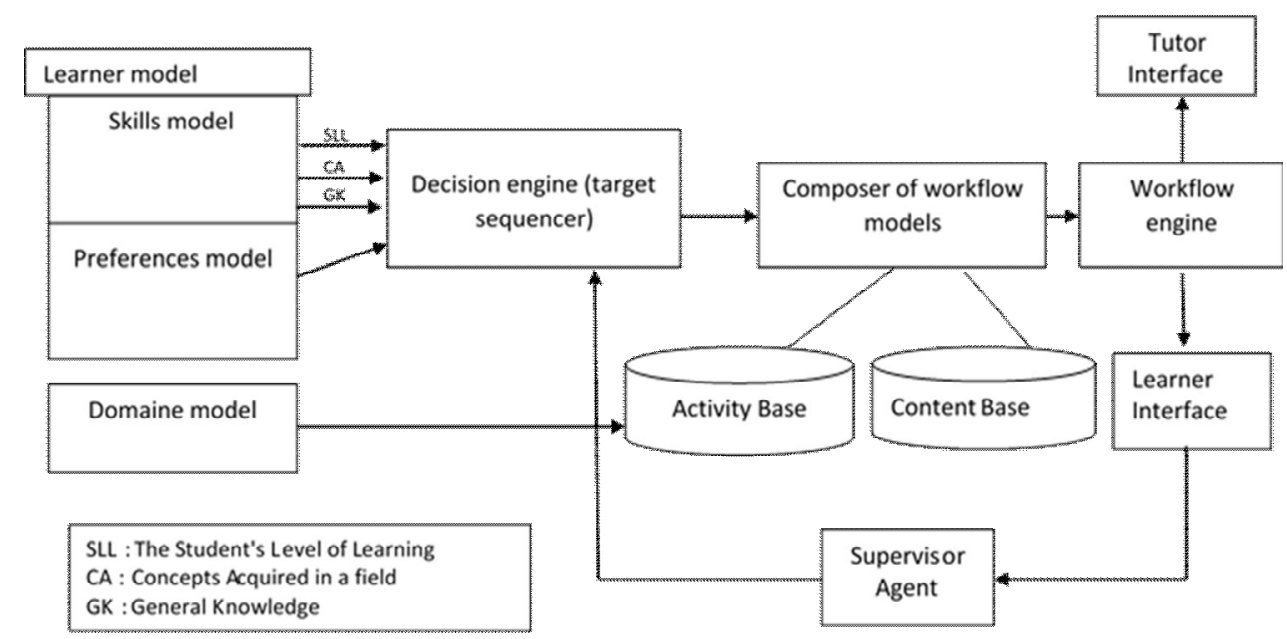

Figure 9: Architecture of the proposed system

\section{Conclusion:}

In this paper, we have begun designing an intelligent tutor system based on adaptive workflows. This was done by first describing a meta-model of the learning process that generates workflow models, and then by modeling the global selflearning process that we will adopt in our system. The second stage involved the design of the learner model and the parameters taken into account in the adaptation process, some of which are exploited in this work, others will be used in our future research. We then defined the outline of our model of teaching domain that we will dig deeper into the mechanisms of its implementation. In the last part of this work, we have developed a model for adapting the learning sequences to the knowledge and preferences of the learner, based on an adaptive workflow engine which permit the intelligent adjustment of didactical activity proposed to the learner and a continuous monitoring and control of the evolution of the learner's abilities by the human tutor in order to have a relevant learning process.

\section{Perspectives:}

In our future work, we will carry out our research in the different modules of the proposed system by digging more on the mechanisms of implementation of the recommended approaches. Concerning the learner's model chosen, which is mainly based on skills and preferences, we try to design it and explore the means of its implantation in order to choose a suitable one to our system. As for the exploitation of adaptive workflows, our research will focus on the improvement of the proposed meta-model in order to predict the automatic generation of self-learning workflows adapted to each learner based on the parameters of his profile. We also try to choose the parameters to be supported by the decision engine and their operations in order to provide automatic adaptation of activities and teaching tasks during a learning sequence. We will discuss the methods of evaluating the learner during and at the end of a pedagogical sequence and how to adapt the parameters of the student's model by using the learner's interactions with the system. 


\section{REFERENCES}

[1] Bruillard Eric 1997. Les machines à enseigner. Chapitre 5 : Des tuteurs intelligents aux environnements interactifs

[2] Hollingsworth, D., \& Hampshire, U. K. (1995). Workflow management coalition: The workflow reference model. Document Number TC00-1003, 19.

[3] MAHDAOUI, L., Boukhedouma, S., \& ALIMAZIGHI, Z. Modélisation de processus e-Learning par les workflows.

[4] Vantroys, T., \& Peter, Y. (2002, November). Un système de workflows flexible pour la formation ouverte et à distance. In Technologies de l'Information et de la Communication dans les Enseignements d'ingénieurs et dans l'industrie (pp. 97104). Institut National des Sciences Appliquées de Lyon.

[5] KORCHI, A., OUGHDIR, L., \& EL IDRISSI, N. E. A. Modélisation de l'apprenant dans un EIAH à base d'ontologie (cas de l'apprenant et son profil) (2015).

[6] ESI, M. M. K. P. (2010). Modélisation basée ontologies pour l'apprentissage interactif-Application à l'évaluation des connaissances de l'apprenant.

[7] Brusilovsky P. « Adaptive hypermedia », User Modeling ans User-Adapted Interaction (1;2), November 2001, p 87-110.

[8] Brusilovsky, P. (2003). Developing adaptive educational hypermedia systems: From design models to authoring tools. In Authoring tools for advanced technology Learning Environments (pp. 377-409). Springer Netherlands.

[9] Felder R.M. and Silverman L.K., 'Learning and Teaching Styles in Engineering Education', Engr. Education, 78(7), 674681,1988

[10] Kolb D.A., LSI Learning-Style Inventory, McBer \& Company, 1985

[11] Paquette, G. (1996). La modélisation par objets typés-une méthode de représentation pour les systèmes d'apprentissage et d'aide à la tâche. Sciences et techniques éducatives, 3(1), 9-42.

[12] Paquette, G. (2002). L'ingénierie pédagogique: pour construire l'apprentissage en réseau. Puq.

[13] Piombo, C. (2007). Modélisation probabiliste du style d'apprentissage et application à l'adaptation de contenus pédagogiques indexés par une ontologie (Doctoral dissertation).

[14] Schreiber, G. (2000). Knowledge engineering and management: the CommonKADS methodology. MIT press.

[15] G. Schreiber, B. Wielinga, J. Breuker. KADS: A Principled Approach to Knowledge-Based System Development. Academic Press, London.

[16] Villanova-Oliver, M. (2002). Adaptabilité dans les systèmes d'information sur le web: Modélisation et mise en oeuvre de l'accès progressif (Doctoral dissertation, Institut National Polytechnique de Grenoble-INPG).

[17] Hnida, M. E. R. I. E. M., Idrissi, M. K., \& Bennani, S. A. M. I. R. (2014). A formalism of the competency-based approach in adaptive learning systems. WSEAS Transactions on Information Science and Applications, 11, 83-93. 